\title{
Los maestros y las maestras de escuela en el Estado institutor: su profesionalización y vinculación a la burocracia del Estado Soberano de Santander, 1870-1885*
}

School Teachers in the Educational State: Their Professionalization and Joining in the Bureaucracy of the Sovereign State of Santander, 1870-1885

Os professores e professoras da escola no Estado pedagogo: sua profissionalização e vinculação à burocracia do Estado Soberano de Santander, 1870-1885

\section{Álvaro Acevedo-Tarazona** (iD) orcid.org/0000-0002-3563-9213 Rolando Humberto Malte-Arévalo*** (iD) orcid.org/0000-0002-2487-6897}

\footnotetext{
Artículo de investigación

Revista Colombiana de Educación, N.7 74. Primer semestre de 2018, Bogotá, Colombia.

Para citar este artículo: Acevedo, A., y Malte, R. (2018). Los maestros y las maestras de escuela en el Estado institutor: su profesionalización y vinculación a la burocracia del Estado Soberano de Santander, 1870-1885. Revista Colombiana de Educación, (74), 19-35.
}

\section{(c) $($ ) (8)

* Este artículo es resultado del proyecto "Desenvolvimiento histórico de la Escuela de Artes y Oficios de Bucaramanga: primer esfuerzo por incorporar la educación técnica en el Departamento de Santander", financiado por la Vicerrectoría de Investigación y Extensión de la Universidad Industrial de Santander, código: 9311, fecha de aprobación: junio de 2014.

** Doctor en Historia, Posdoctorado en Ciencias de la Educación. Profesor titular Escuela de Historia, Universidad Industrial de Santander, Bucaramanga, Colombia. Correo electrónico: acetara@uis.edu.co

*** Magíster en Historia. Miembro del Grupo de Investigación Políticas, Sociabilidades y Representaciones His tórico-Educativas. Docente del Instituto Promoción Social, Bucaramanga, Colombia. Correo electrónico: maltearevalorolando@gmail.com 


\title{
Resumen
}

Este artículo describe y analiza el proceso de profesionalización y de vinculación de los maestros y maestras de escuela al aparato burocrático del Estado Soberano de Santander a partir de la Reforma Instruccionista de 1870. Se argumenta que la puesta en marcha de un sistema de profesionalización de maestros y maestras, así como su vinculación a la burocracia estatal fueron los principales aportes de aquella reforma educativa liberal, dadas las implicaciones institucionales y sociales que trajo consigo, como lo fueron el reconocimiento de un nuevo estatus disciplinar y profesional para la docencia y la aparición de una nueva figura estatal: el maestro de escuela, un aspecto de la Reforma aún no señalado por la historiografía especializada. El estudio se apoya en información primaria de orden oficial, así como en la historiografía que ha abordado el tema de la Reforma para generar una representación histórica del impacto institucional y social de la reforma educativa más importante del siglo XIX colombiano.

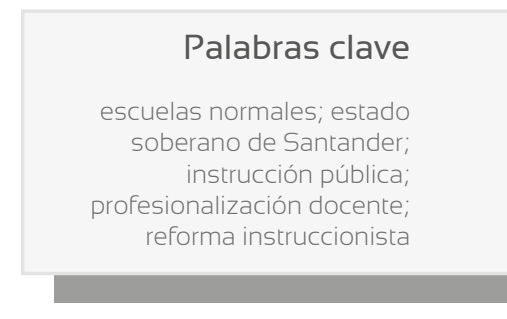

\section{Keywords}

normal schools; sovereign state of Santander; public instruction; teacher professionalization; instructional reform

\begin{abstract}
This paper describes and analyzes the process of professionalization and joining of school teachers in the bureaucracy of the Sovereign State of Santander following the Instructional Reform of 1870 . We argue that the implementation of a teacher professionalization system, as well as their joining in state bureaucracy were the main contributions of the liberal educational reform, given its institutional and social consequences, such as the recognition of the new disciplinary and professional status of teaching and the emergence of a new state figure: the school teacher-an aspect of the reform not yet identified by specialized historiography. The study is based on official primary data, as well as on historiography, which has addressed the subject matter of the reform in order to generate a historical representation of the institutional and social impact of the most important educational reform of the 19th Century in Colombia.
\end{abstract}

\section{Resumo}

Este artigo descreve a analisa o processo de profissionalização e de vinculação dos professores e professoras de escola ao aparato burocrático do Estado Soberano de Santander a partir da Reforma Instruccionista de 1870. Argumenta-se que a implementação de um sistema de profissionalização de professores e professoras, assim como sua vinculação à burocracia estadual, foram as principais contribuições daquela reforma educativa liberal, dadas as implicações institucionais e sociais que trouxe consigo, como o reconhecimento de um novo status disciplinar e profissional para a docência e a aparição de uma nova figura estadual: o professor de escola, um aspecto da Reforma ainda não assinalado pela historiografia especializada. O estudo é sustentado em informação primária de ordem oficial, assim como na historiografia que abrange o tema da Reforma para gerar uma representação histórica do impacto institucional e social da reforma educativa mais importante do século XIX na Colômbia.

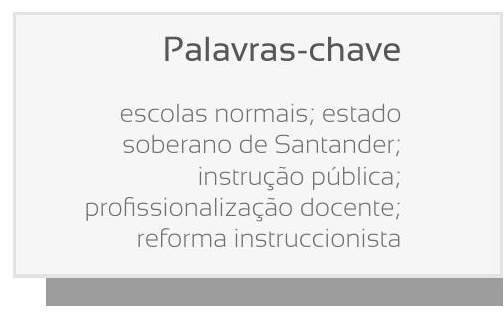




\section{Introducción}

Durante la segunda mitad del siglo xIx, el liberalismo en el Estado Soberano de Santander (Colombia) decidió conferirle al sistema escolar un papel preponderante en el proceso de organización de la república. Su propósito concreto fue convertir a la escuela en la institución encargada de formar a los futuros ciudadanos colombianos, es decir, de difundir entre la población infantil las nuevas reglas del juego político: las reglas del juego democrático (Wilches, 1879). Para alcanzar ese objetivo, e influenciados por las ideas imperantes en Europa y Estados Unidos, mediante el Decreto Orgánico de Instrucción Pública Primaria del 1. e noviembre de 1870 (DOIPP), la principal estrategia adoptada consistió en poner en marcha una reforma educativa que se centrara en la ampliación de la cobertura escolar, en el ofrecimiento de más y mejores medios educativos, en la renovación de los métodos de enseñanza y, sobre todo, en la formalización del proceso de profesionalización del cuerpo de maestros y de su vinculación al aparato estatal. Así, además de la prensa y el club político -como lo ha señalado Gilberto Loaiza Cano (2007) en su estudio sobre la sociabilidad política del siglo xix colombiano, demostrando que la prensa, el club político y la escuela fueron los principales espacios de sociabilidad política-, la reforma de las instituciones educativas y la creación de un cuerpo estatal moderno de maestros de escuela, el liberalismo establecía una estrategia oficial de difusión de los principios de la democracia moderna, con lo cual propiciaba un cambio institucional y social inédito.

Hasta el momento, los estudios históricos acerca del fenómeno aludido (Álvarez Gallego, 1995; Báez, 2004; Báez, 2005; González, 2005; Loaiza Cano, 2007) se han centrado en el análisis de sus aspectos más evidentes: 1) la manera en que la reforma organizó una serie de instituciones educativas nunca antes vistas en el país, como la Universidad Nacional o las escuelas normales; 2) la feroz resistencia de la Iglesia católica y el Partido Conservador contra este proyecto de corte liberal, ya que declaraba la laicidad en materia educativa y su obligatoriedad; y 3) su importancia cultural al orientar el proyecto de nación colombiano en la ruta de la modernidad, gracias a la implementación del método pedagógico pestalozziano y de sus diversas técnicas de disciplinamiento. En resumen, y si bien las investigaciones mencionadas señalan que la reforma educativa de 1870 fue un fenómeno político que trascendió los márgenes mismos del ámbito escolar e hizo evidente la multiplicidad de nexos políticos, sociales y culturales que atraviesan al hecho educativo, quedan por dilucidar sus implicaciones institucionales y sociales, principalmente el establecimiento de un proceso de profesionalización de maestros y de su vinculación a la burocracia estatal. No cabe duda de que la reforma fue una estrategia diseñada e implementada por el Olimpo Radical para justificar el nuevo 
orden político, es decir, para construir y mantener el orden republicano; y queda claro también que la reforma de 1870 fue el primer proyecto educativo colombiano llevado a la práctica, pero ¿qué efectos trajo consigo esta reforma y de qué clase fueron?

El objetivo de este artículo es describir y analizar el proceso mediante el cual la reforma propició la apertura de la enseñanza como espacio sociopolítico. La idea central indica que los principales aportes de la reforma educativa liberal consistieron en propiciar el reconocimiento de un nuevo estatus disciplinar y profesional para la enseñanza y en convertir al maestro de escuela en una nueva figura del entramado estatal, todo esto a través de la organización de un sistema de profesionalización de maestros y de una serie de estrategias que permitirían su vinculación al aparato burocrático. Se arguye, por consiguiente, que en términos analíticos, principalmente del nuevo institucionalismo, la propuesta reformadora del liberalismo de la segunda mitad del siglo xix se proponía instituir, desde la escuela, las reglas del juego democrático. Como se verá en la presente investigación, esta tarea fue llevada a cabo a través del DoIPp y materializada en los maestros de escuela como funcionarios profesionales.

Para sustentar estas ideas, en el presente estudio proponemos un análisis histórico neoinstitucional, tanto del contenido de la reforma como de su aplicación, de manera que se pueda sacar a flote la relevancia de los cambios sociales e institucionales propiciados por el liberalismo en el Estado Soberano de Santander. Este es un enfoque teórico-metodológico que proviene de distintos sectores de las ciencias sociales. En la economía política, el neoinstitucionalismo fue una reacción contra la teoría conductual, enfoque que interpretaba "la conducta económica y política colectiva como una consecuencia agregada de la elección individual" obviando abiertamente el contexto social (Powell y Dimaggio, 2001, p. 34). En el sector de la sociología política, surgió entre quienes desconfiaban del funcionalismo porque consideraban que este no ayudaba a "comprender las interconexiones duraderas entre la organización política, la economía y la sociedad" (pp. 34-35). Finalmente, una tercera corriente neoinstitucionalista se originó en tres campos disciplinares: la microsociología, la historia social y los estudios culturales, disciplinas que, a diferencia del determinismo funcionalista, sugirieron que "las referencias individuales y categorías básicas del pensamiento como la personalidad, la acción social, el Estado y la ciudadanía son conformadas por fuerzas institucionales" (p. 35).

Debido a esta múltiple procedencia histórica, la definición que los distintos sectores dan del concepto de institución no es unívoca. En el campo de la economía, por ejemplo, los investigadores proponen que las instituciones económicas son mecanismos a través de los cuales las sociedades pueden hacer cumplir los acuerdos pactados entre los individuos o las organizaciones, eliminando al máximo las incertidumbres propias 
de todo intercambio económico. En consecuencia, entienden por instituciones tanto las "estructuras de gobierno" como los "acuerdos sociales ajustados para reducir al mínimo los costos de transacción" económica (Powell y Dimaggio, 2001, p. 42).

En el campo de la política, son dos las metas que el neoinstitucionalismo quiere alcanzar. Por una parte, pretende entender la forma en que se originan y funcionan las instituciones políticas nacionales, y por otra, la forma en que se organizan las relaciones internacionales. A la primera se le da el nombre de teoría positiva, y a la segunda, el de teoría de los regímenes. En ambas teorías las instituciones son vistas como estructuras estabilizadoras de la vida política, ya que suponen que las reglas legislativas eliminan la inestabilidad generada por los costos de transacción del intercambio político -a nivel nacional-y tienden a buscar la cooperación entre las naciones -a nivel internacional-. También en ambos casos se supone una sociedad en la que los actores, para alcanzar y lograr mayores beneficios, crean instituciones que regulan la vida en sociedad tanto en la nación como en el mundo entero. Por estas razones definen a las instituciones como "conjuntos de principios, normas, reglas y procedimientos de toma de decisiones implícitas o explícitas en torno a los cuales convergen las expectativas de los actores" sociales y políticos (Powell y Dimaggio, 2001, pp. 38-42).

Finalmente, en el ámbito de la sociología, la teoría neoinstitucional tiene como meta entender el origen, la estructura y los mecanismos del funcionamiento de las diferentes clases de organizaciones y el papel de las instituciones en su configuración. Desde esta perspectiva, Ronald J. Jepperson (2001) manifiesta que la mayoría de los especialistas dan al vocablo institución tres clases de significado: algunos lo usan para referirse a las asociaciones; hay quienes identifican la institución con "Ios efectos ambientales" (p. 193), y otros usan el término como referente de los "efectos culturales o históricos" (p. 195). A su modo de ver, este tipo de equivocaciones debe corregirse para dar solidez a los conceptos. Por esta razón, propone que el contenido semántico del término institución sea principalmente disociado del concepto organización. Una institución -propone- debe ser entendida como un orden o patrón social que ha logrado consolidarse como principio rector del comportamiento social. Mientras que la organización debe ser vista como el agregado de individuos con fines o intereses comunes, con funciones implícitas reconocidas, apoyadas y "legitimadas públicamente" por unas organizaciones políticas más amplias, como el Estado y la sociedad civil (Jepperson y Meyer, 2001, pp. 263-265).

Pues bien, es aquí donde esta investigación encuentra su sustento teórico. En efecto, en el presente estudio se usa el concepto de instituciones para hacer referencia a los "sistemas de programas o de gobierno socialmente construidos y reproducidos rutinariamente" con el fin de ordenar 
el comportamiento social (Jepperson, 2001, p. 201). Por organizaciones se nombra a aquellos grupos de individuos que han sido creados para alcanzar objetivos comunes, que son legitimados por una organización mayor de tipo político, que regulan su funcionamiento a través de normas explícitas con las cuales obtienen, además, identidad y que buscan su propia perpetuación en el tiempo así como su crecimiento en el espacio (Jepperson y Meyer, 2001, p. 266).

Apoyado en esta conceptualización, así como en documentación primaria y en historiografía, el presente estudio se aproxima a la historia social e institucional del Estado Soberano de Santander, con el fin de evidenciar la aparición de los maestros modernos como actores sociales y políticos de primer orden en la sociedad que apenas iniciaba el trasegar hacia la modernidad. En este sentido, el estudio muestra, en apartados específicos, primero, cómo se instituyó el ramo de la instrucción pública y, segundo, cómo se llevó a cabo el proceso de vinculación de los maestros de escuela a la burocracia del Estado Soberano de Santander.

\section{La transformación de la instrucción pública en rama de la administración gubernamental}

La reforma instruccionista de 1870 fue un programa político puesto en marcha por el Gobierno central de los Estados Unidos de Colombia y aplicado por el Gobierno federal del Estado Soberano de Santander ${ }^{1}$. Dicho programa ponía bajo el amparo de ambos Gobiernos la organización, la dirección, el control y la financiación de la instrucción pública. Para llevar a cabo el proyecto, la principal tarea de la reforma consistió en convertir a la instrucción pública en una rama de la administración gubernamental. Este proceso fue ordenado tanto por leyes nacionales como federales. Entre las primeras, encontramos la Ley xxvII del 30 de mayo de 1868, la Ley Lxxxı del 2 de julio de 1870 y el Decreto Orgánico de Instrucción Pública Primaria del $1 .{ }^{\circ}$ de noviembre del mismo año. Mediante la Ley xxvı se ordenó, entre otras cosas, la "injerencia del Gobierno general en el ramo de instrucción pública" (Anzola, 1933, p. 348) con el objeto de: 1) sostener a la Universidad Nacional fundada en septiembre de $1867 ; 2$ ) establecer en la capital de la Unión, escuelas normales para la formación de maestros; 3) crear en todo el territorio nacional varias escuelas públicas rurales y urbanas de instrucción primaria, y 4) unificar el sistema de instrucción pública primaria de toda la nación. Por su parte, con la Ley Lxxxı de julio de 1870 (Mejía, 2007, p. 389) se autorizó al Ejecutivo para que fundara

1 Entre 1863 y 1886 Colombia había adoptado el régimen federativo. Su nombre era Estados Unidos de Colombia y suscribía siete Estados Soberanos, entre ellos el de Santander. 
las escuelas normales en las capitales de los Estados. Finalmente, por intermedio del DolpP, se reformó y se reglamentó pormenorizadamente el sistema de instrucción pública para toda la nación (Diario Oficial, 1870).

Del mismo modo procedieron los poderes ejecutivo y legislativo del Estado Soberano de Santander al crear la Ley xxxvı del $1 .^{\circ}$ de diciembre de 1868 y el Código de Instrucción Pública del Estado (CIP). Mientras que por la primera se ordenó al gobierno hacerse cargo de las escuelas de los distritos municipales (Gaceta de Santander, 1870, n. ${ }^{\circ} 664$, año xxı, p. 501), con el CIP se organizó el sistema de educación que regiría en el territorio santandereano. Su mayor logro fue configurar la instrucción como un todo dividido en "tres grandes ramos" (Códigos Legislativos, s. f., p. 528): la enseñanza, la inspección y la administración, cada uno de ellos con sus respectivas funciones.

Para sostener financieramente esta nueva entidad gubernamental, la reforma utilizó tanto los dineros que proveía el Gobierno central -al aceptar la Ley xxVII del 30 de mayo de 1868, con la cual este se hacía cargo del sostenimiento de una buena cantidad de instituciones educativas establecidas en los Estados- como los ingresos que recaudaba el Estado Soberano a través de las rentas de aguardiente y degüello, y a través de las contribuciones especiales como las establecidas en la Ley xvı de 1867, que grababan los contratos de instrucción con un cargo de un décimo de peso, o la Ley xxvIII de 1867 que estableció un impuesto al consumo de licores extranjeros. Por último, se recurrió a las multas para aquellos padres de familia que no enviaran a sus hijos a las escuelas.

Estos y otros gravámenes aplicados durante el periodo liberal permitieron al Estado mejorar sus finanzas. Atrás quedaba la desdichada situación económica presentada entre 1859 y 1862, cuando los ingresos del Estado escasamente alcanzaban "para atender a sus gastos ordinarios" (Gaceta de Santander, 1859, n. ${ }^{\circ}$ 76, año II, p. 321), según decían los gobernantes. En contraste con aquella época, momento en el cual el Estado pudo con dificultad recaudar $\$ 70.200$, entre 1863 y 1885, el recaudo efectivo creció 8,5 veces (Malte, 2010). Esta situación permitió que la financiación de la instrucción con fondos públicos fuera todo un acontecimiento a partir de 1869 y se mantuviera con el paso de los años.

La reforma contó entonces con recursos financieros que le permitieron fomentar el aumento de la cobertura escolar y el perfeccionamiento del servicio con mejores maestros y nuevos materiales didácticos. Pese a que no se puede afirmar que todo el sector educativo creció de manera sostenida, se debe señalar que hubo logros significativos. La cobertura, por ejemplo, tuvo un considerable crecimiento. A diferencia de los años anteriores, entre 1868 y 1885, los Gobiernos pudieron mantener en funcionamiento por lo menos dos instituciones educativas por distrito, 
e incluso entre 1871 y 1880, en la mayoría de los distritos, hubo más de dos entidades escolares, varias de ellas dedicadas a la formación profesional, como el Colegio Universitario de Santander o las escuelas normales.

Como entidad estatal, mediante la reforma instruccionista los Gobiernos de la época lograron reestructurar la instrucción pública. Para los reformadores, el término Instrucción Pública hacía referencia a una organización configurada por dos elementos diferenciados pero íntimamente relacionados: la estructura burocrática, por un lado, y la estructura escolar, por otro. En consecuencia, la Instrucción Pública fue tanto una rama de la burocracia gubernamental (con funcionarios, reglamentos, funciones y estímulos) como el conjunto de entidades educativas debidamente organizadas (con métodos y contenidos de enseñanza, reglamentos y sistema de promoción).

Desde el punto de vista estrictamente administrativo, la reforma creó una estructura burocrática conformada por una serie de entidades encargadas de la dirección, la financiación y la vigilancia del ramo. Estas entidades abarcaban distintos marcos político-administrativos. En el nivel central y bajo la tutela de la Secretaría del Interior y de Relaciones Exteriores, se encontraba la Dirección General de Instrucción Pública; en el marco del Estado Soberano de Santander esta tarea la cumplía la Dirección de Instrucción Pública; en el nivel local, las jefaturas departamentales y distritos, el control lo ejercían los concejos departamentales y las comisiones de vigilancia.

En referencia a la organización escolar², la instrucción pública ya abarcaba los elementos pedagógicos y de gestión educativa que le son propios al sistema escolar moderno. En el lenguaje de la época, para definir este sistema se utilizó el concepto de enseñanza, una designación mediante la cual se agruparon los diferentes aspectos de un modelo pedagógico instruccionista ${ }^{3}$. Esto implicó una estructura escolar con un objetivo concreto (formar ciudadanos), con principios pedagógicos específicos (la pedagogía de Pestalozzi), con una organización propia para los contenidos curriculares -Historia Patria, Ciencias Naturales, Lectura, Escritura, Aritmética, Cívica y Moral-,

2 Las organizaciones, según lo ha postulado la teoría sociológica y administrativa, son "unidades sociales (o agrupaciones humanas) deliberadamente construidas o reconstruidas para alcanzar fines específicos". Las corporaciones, los ejércitos, las escuelas, los hospitales, las iglesias o las prisiones son ejemplos de organizaciones (Etzioni, 1965, p. 4-5; Jepperson y Meyer, 2001, p. 263-265).

3 Como se sabe, toda educación tiene como problema esencial definir el tipo de ser humano y sociedad que quiere edificar y toda respuesta a esta problemática constituye una teoría pedagógica. En su construcción, las teorías pedagógicas parten de unas concepciones acerca del ser humano y de la sociedad, que les permitirán estructurar el proceso educativo. Esa estructuración es lo que se denomina modelo pedagógico. En otras palabras, en "un modelo pedagógico se establecen los lineamientos" que guiarán el proceso educativo, o si se prefiere, los componentes que lo estructurarán, que no son más que los propósitos educativos, los saberes o contenidos, la forma de organización de esos contenidos, el método o metodología para transmitirlos, los recursos didácticos y la forma de evaluar la eficacia del aprendizaje (De Zubiría, 1997, p. 38-41). 
con un determinado tipo de materiales didácticos para facilitar el aprendizaje (libros, objetos de laboratorio, citolegias, pizarras, entre otros), y con técnicas de evaluación y disciplinamiento encaminadas no solo a valorar el desarrollo académico de los estudiantes sino a corregir su comportamiento.

Como puede observarse, a través del DOIPP y el CIP, principalmente, la reforma instituyó una serie de medidas legales con las cuales se pretendió normalizar la conducta social de los habitantes del Estado Soberano de Santander. Para ello, la reforma reestructuró la instrucción pública, en primer lugar, mediante la creación de un aparato burocrático instruccionista, y en segundo lugar, a través de la creación de las organizaciones escolares. Ambos aspectos pusieron en marcha un sistema moderno de instrucción pública.

\section{Maestros de escuela: nuevos funcionarios estatales}

La vinculación de los maestros de escuela a la burocracia del Estado Soberano de Santander se dio a través del proceso de creación del funcionariado profesional ${ }^{4}$ del ramo instruccionista. Este, tal como sucedió con la administración de la enseñanza, también fue instituido por el DOIPP y el CIP. Se trataba, en consecuencia, de un proceso racional y moderno que consideraba que el buen funcionamiento del sistema dependía de la calidad profesional de los maestros que lograra vincular al aparato burocrático, razón por la cual la reforma creó unas organizaciones de formación especializada, estableció los términos específicos de contratación laboral y organizó al gremio profesional.

Aunque todos estos aspectos se establecieron simultáneamente, es necesario analizarlos de manera independiente.

\section{Instituciones de formación}

El establecimiento de un sistema especial de formación de maestros fue una tarea central de la reforma instruccionista, puesto que, hacia mediados del siglo xIx, en Colombia tampoco estaban generalizados los estudios

4 El término funcionario profesional se entiende según la concepción de Max Weber, para quien el funcionariado profesional está conformado por todas las personas incluidas en una administración burocrática -es decir en un tipo de ordenación cuya dominación es de carácter racional (Weber, 1974), tal como sucede en el Estado moderno o en las empresas capitalistas-, que actúan bajo los siguientes principios: se proponen, personalmente libres, cumplir con los deberes objetivos de su cargo; obedecen una jerarquía administrativa rigurosa; tienen competencias rigurosamente fijadas en virtud de un contrato; cuentan con una calificación profesional que fundamenta su nombramiento; reciben una retribución en dinero con sueldos fijos; ejercen el cargo como única o principal profesión; tienen ante sí una carrera, o perspectiva de ascensos y avances por años de ejercicio; trabajan con completa separación de los medios administrativos y sin apropiación del cargo, y están sometidos a una rigurosa disciplina y vigilancia administrativa (Weber, 1974). 
formales relacionados con la profesión pedagógica. No se debe creer, sin embargo, que con anterioridad a la reforma liberal el país no había emprendido proyectos similares. En efecto, el primero en proponer la fundación de escuelas normales fue Mariano Ospina Rodríguez, ministro del Interior durante el Gobierno conservador de Pedro Alcántara Herrán, en 1844. Su idea había consistido en reemplazar el sistema de instrucción lancasteriano instaurado por el General Francisco de Paula Santander en 1821 -que consistía en convertir a los alumnos más adelantados de la escuela en maestros de sus propios compañeros- por uno que separaba la formación de los maestros de la instrucción de los alumnos. Fue entonces cuando apareció en el país la primera escuela especializada en la formación para la enseñanza. Aquel establecimiento fue fundado en Bogotá bajo el nombre de Escuela Normal; estuvo a cargo de José María Triana (Herrera y Low, 1990; Zuluaga, 2011, p. 41-42) y si bien no arrojó los mejores resultados fue el antecesor de las escuelas normales que los reformadores liberales instaurarían a partir de 1870.

Fueron entonces los liberales de la segunda mitad del siglo xix quienes mayor impulso les imprimieron a estas organizaciones educativas. Para ello pusieron en marcha una idea sugerida en 1865 por José María Villamizar Gallardo, quien por entonces ejercía como presidente del Estado Soberano de Santander. La propuesta presentada a la Asamblea Legislativa del Estado recalcó que para solucionar el problema de la falta de maestros competentes e impulsar un programa masivo de alfabetización era prioritario crear escuelas normales (Villamizar, 1865). Como la proposición no se pudo aplicar debido a las dificultades financieras del Estado, Villamizar decidió llevar al Senado de la República una nueva versión de su idea (Cadena, 1874). En esta ocasión, sugirió responsabilizar al Gobierno central tanto de la fundación de aquellas instituciones como de su sostenimiento. El Congreso, aunque no de manera inmediata, aceptó su propuesta y promulgó las leyes con las cuales se hizo efectiva la fundación de escuelas normales.

La primera escuela de este tipo fue fundada en Bogotá mediante la Ley xxvII del 30 de mayo de 1868. En el caso santandereano, la primera normal fue establecida por la Ley xiv del 18 de octubre de 1871, pero se trataba de una institución para varones. Dos años después, el 27 de julio de 1873, se fundó la Escuela Normal de Institutoras del Estado. Le siguieron la Escuela Normal Nacional de Institutoras de Bucaramanga (establecida el 13 de enero de 1875), la Escuela Normal de Artes y Oficios (creada por la Ley xxxv del 9 de octubre de 1876) y la Escuela Normal de Institutores del Estado (creada mediante la Ley $v$ del 30 de septiembre de 1882). Pese a que todas estas instituciones fueron fundadas legalmente, solo las tres primeras tuvieron existencia real. La Escuela Normal Nacional de Institutores inició actividades el 2 de octubre de 1872 en El Socorro, la capital del Estado. La Normal de Institutoras del Estado entró en funcionamiento entre octubre 
y noviembre de 1873, y la Escuela Normal Nacional de Institutoras de Bucaramanga en febrero de 1875. Estas tres instituciones permanecieron abiertas durante el resto del periodo federal, pese a que el desarrollo normal de sus actividades se vio afectado por los conflictos políticos que marcaron la época. Una de ellas, la Escuela Normal de Institutoras de Bucaramanga, sigue aún en funcionamiento.

El proceso de formación docente impartido en estas instituciones se apoyaba en las siguientes estrategias: 1) aplicación de una serie de reglamentos institucionales que permitieran vigilar y guiar el comportamiento de los alumnos maestros; 2) desarrollo de clases teóricas y prácticas de pedagogía para transmitir y ejercitar los saberes especiales de la profesión; 3) realización de exámenes de graduación como instancia que separaba al aprendiz del profesional; y 4) realización de actividades encaminadas a fortalecer el amor patrio, como las conmemoraciones de las fiestas de la nación.

Los reglamentos le permitirían al proceso de enseñanza organizar y dirigir las actividades formativas, así como seleccionar los alumnos que tuvieran los caracteres propios del maestro ideal. El reglamento autorizaba la expulsión de aquellos estudiantes que manifestaran mal carácter, falta de aptitudes, desaplicación, salud delicada, o que claramente dejaran conocer que no poseían "ninguna vocación para la pedagogía" (Gaceta de Santander, 1882, n. ${ }^{\circ}$ 1467, año xxIv, p. 92). La presión a la cual estuvieron sometidos debió ser fuerte. Las subdirectoras de las normales, por ejemplo, estaban encargadas de llevar un "registro diario de la conducta" (p. 92) de sus alumnas y de remitir unos informes mensuales en los que se especificaba su grado de "aprovechamiento" (p. 92). Al contar con una técnica de registro de las conductas, el modelo de formación lograba configurar un cuadro descriptivo ${ }^{5}$ de los progresos o retrocesos que cada una de las estudiantes experimentaba a lo largo de su proceso de formación, de manera que se pudiera contar con información suficiente para permitirle al sistema seleccionar a las mejor capacitadas para el oficio.

Como puede observarse, la reforma instruccionista en efecto instituyó un tipo de organización especializada en la formación de maestros. Dichas organizaciones, en concordancia con los principios pedagógicos del momento, contaban con una estructura curricular especial, cuyo objetivo era dotar a sus estudiantes de los conocimientos y las habilidades que les permitirían dirigir adecuadamente los procesos de instrucción. Como cualquier otra organización moderna, las escuelas normales establecidas por la reforma contaban con una estructura administrativa racional, recibían fondos estatales, tenían fines específicos, involucraban un personal

5 El sistema de instrucción pública liberal creó una variada serie de cuadros para ejercer control. Había cuadros de listas de estudiantes, de asistencia, de notas, de conducta (Álvarez, 1995). 
especializado, eran objeto de vigilancia social y gubernamental y lograron mantenerse a lo largo del tiempo. En consecuencia, la existencia de estas organizaciones evidencia que las instituciones creadas por la reforma instruccionista propiciaron cambios sociales en el interior del Estado Soberano de Santander, entre los cuales el principal fue la transformación de la enseñanza en una profesión moderna.

\section{Oficialización de la enseñanza}

Además de convertir la enseñanza en una profesión, la reforma también creó una estrategia especial para insertar a los maestros a la burocracia estatal, como ya se dijo. Esta estrategia consistió en reglamentar la contratación oficial de los institutores en concordancia con los principios administrativos modernos: contrato escrito, manual de funciones y pago de salarios acordes con la función y la formación de los trabajadores. La contratación y el pago de salarios los determinó la Ley xxxIII del 8 de octubre de 1870, con la cual la reforma lograba solucionar uno de los principales obstáculos al desarrollo de la instrucción pública. En efecto, antes de la reforma, el salario de los maestros lo pagaban directamente los padres de familia o las autoridades locales, lo que acarreaba graves dificultades en el desarrollo de las actividades educativas, debido a la irregularidad de los pagos (Malte, 2010, p. 42-45). En consecuencia, se propusieron medidas legislativas que lograran neutralizar los efectos negativos de aquella penosa situación, ordenando, principalmente, que los sueldos de los maestros fueran "fijados por el Superintendente de la Instrucción Pública" (Gaceta de Santander, 1870, n. ${ }^{\circ} 661$, año xxı, p. 486) y cancelados por el Gobierno.

Establecido el derrotero, la reforma procedió a estructurar la manera en que se llevarían a cabo tanto la contratación como el pago de los salarios de los institutores que entrarían al servicio del Estado. La contratación era un evento público efectuado ante las autoridades gubernamentales del ramo de instrucción. En el contrato firmado se estipulaban los derechos y deberes del funcionario, así como las medidas disciplinarias que se adoptarían si incurría en algún tipo de falta.

Para calcular el salario de cada docente, el Doipp creó una tabla de salarios con base en el nivel profesional de los maestros, el tipo de institución escolar que dirigían, el cargo, el género y la categoría político-administrativa del lugar de trabajo. Así por ejemplo, en 1870 los salarios de los maestros fluctuaban entre los $\$ 192$ y los $\$ 480$ anuales, según se tratara de un maestro graduado y nombrado para una escuela elemental local, o de uno graduado y contratado para una escuela superior de un distrito capital (Códigos Legislativos, s. f.; Gaceta de Santander, n. ${ }^{\circ} 1122$, año xIx, 1877). El mismo año, las maestras interinas, pues aún no se contaba con maestras graduadas, ganaban $\$ 120$ anuales, pero a partir de 1876 , cuando 
ya se contaba con una primera generación de institutoras normalistas, el Estado empezó a cancelarles entre $\$ 480$ y $\$ 600$ por año. Este monto era igual al que ganaban los maestros de una escuela local y $\$ 240$ menos que el sueldo de un maestro de un distrito capital.

En comparación con el periodo anterior a la reforma, los nuevos salarios eran muy superiores. En aquella época los maestros ganaban, a lo sumo, entre $\$ 16$ y $\$ 80$ anuales, pero a partir de la reforma sus salarios empezaron a equipararse con los sueldos de los demás funcionarios del Estado, como los escribientes o secretarios de las Jefaturas Departamentales, quienes obtenían sueldos cercanos a los $\$ 200$ y $\$ 550$ por año. Con esta medida los maestros lograban posicionarse entre el funcionariado medio. De hecho, cuando la reforma llevaba casi seis años de aplicación y antes de la guerra civil de 1876-1877, los maestros Ilegaron a ganar tanto como un secretario o un juez civil: cerca de unos $\$ 400$ o $\$ 720$ anuales si se trataba de maestros de primaria, o tanto como un jefe departamental si se trataba de un maestro de escuela normal, esto era, unos $\$ 1.200$ por año.

Con la reforma instruccionista, por consiguiente, los maestros de escuela quedaban ubicados en la categoría media de la burocracia estatal. Su inserción en el entramado del Estado era un proceso legal, que seguía, por ende, los principios racionales de la administración estatal moderna: un contrato suscrito entre un individuo -el ciudadano moderno- y las autoridades de la asociación política a la que este pertenecía. Tal como sucedía con el contrato, el salario de estos nuevos funcionarios también fue reglamentado según los principios burocráticos. Los datos históricos indican, de hecho, que el cálculo salarial dependía de una estrategia racional que tenía en cuenta la formación, el lugar en el que se ejercería la función, el género del funcionario y el tipo de organización escolar en la que se desempeñarían las labores.

Se trataba, pues, de una medida institucional que traía consigo importantes efectos sociales. Por una parte le confería prestigio a un oficio poco demandado antes de 1870; y por otra, les abría las puertas de la vida laboral a las mujeres, al ofrecerles la posibilidad de desempeñarse como maestras de escuela. En efecto, el sistema les otorgaba a las maestras un reconocimiento social y económico diferente al que les confería el rol doméstico. Su función laboral era de hecho equiparable al que recibían los maestros con preparación profesional similar. Eran contratadas y recibían salario del Estado y, como los hombres, también debían seguir el reglamento de la función escolar.

En síntesis, con esta medida la reforma instruccionista instituyó la función de la enseñanza convirtiéndola en una más de las acciones del Estado. Esto se llevó a cabo a través de la oficialización de los procesos de contratación y el pago de salarios, hechos que derivaron en la inserción de los maestros de escuela en el entramado burocrático del Estado Soberano de Santander.

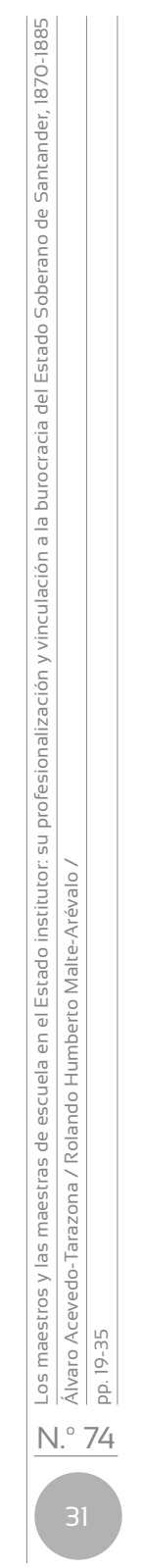




\section{Sociedades de institutores}

La vinculación de los maestros de escuela al aparato burocrático fue complementada con la creación de las sociedades de institutores. Aunque hubo en Santander una asociación de maestros conformada voluntariamente, la mayoría de las sociedades de institutores durante el siglo xix fueron fruto de la acción gubernamental. Esta implementación educativa también fue efecto de la reforma instruccionista. Tanto en el CIP como en el Dolpp se ordenó a los maestros ingresar a estas sociedades. Con algunas diferencias, ambas normas señalaban, en primer lugar, que estarían conformadas no solo por los maestros sino también por los miembros de los Consejos de Instrucción, por los ciudadanos que defendieran la causa instruccionista - "amigos de la educación", decían las normas (Códigos Legislativos, s. f., arts. 121-122; Diario Oficial, número extraordinario, 1870, arts. 136-137)y por los alumnos adelantados; en segundo lugar, que serían establecidas en los departamentos de cada Estado Soberano y presididas por el Director de la Escuela Normal Nacional.

Las Sociedades de Institutores le permitían a la Instrucción Pública cumplir con dos tareas centrales: controlar a los miembros del ramo y fortalecer la profesión desde el punto de vista pedagógico. Ambas tareas se llevaron a cabo a través de un reglamento especial incorporado tanto en el CIP como en el Doipp. En términos de la época, sus finalidades eran: primero, "sostener el honor de la profesión, haciendo que los Institutores públicos [fuesen] el modelo de los buenos ciudadanos" (Códigos Legislativos, s. f., art. 124; Diario Oficial, número extraordinario, 1870, art. 138); segundo, "trabajar en la perfección de los métodos y textos de enseñanza" (Códigos Legislativos, s. f., art. 124; Diario Oficial, 1870, art. 138) y tercero, "dirigir y auxiliar a los maestros cuyas habilidades no estaban suficientemente ejercitadas" (Códigos Legislativos, (s.f.), art. 124; Diario Oficial, 1870, art. 138). Todas estas acciones se desarrollaban en jornadas complementarias de trabajo programadas una vez al mes a manera de reuniones, cursos o lecciones.

Mientras que la función fiscalizadora de las asociaciones les autorizaba, incluso, destituir a "cualquier Director o Subdirector de escuela que por su conducta [fuera catalogado] indigno de su alto magisterio" (Códigos Legislativos, s. f., art. 124; Diario Oficial, número extraordinario, 1870, art. 138), su función formativa les permitía acreditar conocimientos pedagógicos. No fueron pocos, en consecuencia, quienes acudieron a estas asociaciones para entrar en contacto con los métodos modernos de instrucción, y quienes gracias a ellos pudieron habilitarse para el ejercicio de la enseñanza (La Escuela Primaria, n. ${ }^{\circ} 1-42$, año I, 1871, 1872). 
La función institucional de estas organizaciones, finalmente, consistió en fomentar entre la comunidad en general las ideas instruccionistas que imbuían a la reforma. Si bien los miembros principales de las sociedades de institutores eran los maestros de escuela, con la creación de la categoría de miembros correspondientes, la reforma quiso aumentar el número de adeptos al ideario liberal o "amigos de la educación" (La Escuela Primaria, n. ${ }^{0} 1$, año I, 1871, p. 5), es decir, ciudadanos que apoyaran las políticas instruccionistas del Gobierno.

Las asociaciones del gremio de la enseñanza fueron, por consiguiente, la estrategia que complementaría el proceso de inserción de los institutores en el aparato burocrático del Estado Soberano de Santander. Tal como sucedió con las demás estrategias, la creación del gremio de la enseñanza fue también un mecanismo instituido por la reforma instruccionista y por la mentalidad liberal de sus promotores.

\section{Conclusiones}

Del panorama histórico descrito y analizado en este artículo puede concluirse que la reforma instruccionista aplicada en el Estado Soberano de Santander a partir de 1870, más allá de sus logros o deficiencias en materia de cobertura, fue una medida política que generó cambios sociales e institucionales. Al respecto, y en términos analíticos, debe resaltarse que, en primer lugar, la reforma posibilitó la apertura de un nuevo espacio de participación pública al instituir la enseñanza como una más de las dependencias del aparato burocrático. En segundo lugar, no debe perderse de vista que este proceso de burocratización de la enseñanza se llevó a efecto a través de medidas de orden racional, es decir, siguiendo el cauce de las acciones propias de la administración pública moderna. De ahí el que la instrucción pública fomentara el proceso de profesionalización de la enseñanza -creado, planeado y dirigido a los maestros de escuela a través de sendas organizaciones de formación-, creara las entidades de administración que ejercerían el control del ramo de instrucción e instaurara normas encaminadas a regular la contratación, el pago de salarios y la agremiación de los maestros de escuela. Los datos históricos en efecto muestran que las organizaciones de formación existieron: en ellas recibieron título oficial 93 maestros y 66 maestras entre 1874 y 1882 , estos institutores e institutoras fueron vinculados a las escuelas del Estado Soberano, allí ejercieron su función incluso hasta después de haber caído el régimen liberal, todos ellos fueron contratados según las disposiciones legales y, en general, el mantenimiento del ramo contó con apoyo financiero gubernamental, sustentado por fondos provenientes tanto del Gobierno central como del federal. 


\section{Referencias}

\section{Fuentes primarias}

\section{Periódicos}

Gaceta de Santander

Años:

1859 , n. ${ }^{\circ} 76$, año II.

1870, n. ${ }^{\circ} 661$, año XII; n. ${ }^{\circ} 664$, año XII.

1877, n. ${ }^{\circ} 1122$, año XIX.

1882, n. ${ }^{\circ} 1467$, año XXIV.

La Escuela Primaria: periódico oficial de Instrucción Pública del E. de Santander

Años:

1871, n. ${ }^{\circ} 1-6$, año I.

1872, n. ${ }^{\circ} 7-42$, año I.

\section{Otras publicaciones}

Anzola, N. (Dir.). (1933). Codificación Nacional de todas las leyes de Colombia desde el año de 1821, hecha conforme a la Ley 13 de 1912. Tomo XXII, años 1867-1868. Bogotá: Imprenta Nacional.

Cadena, N. (1874). Informe del presidente del Estado de Santander a la Asamblea Legislativa en 1874. Socorro: Imprenta del Estado.

Códigos legislativos del estado de Santander. (1870). Edición oficial, tomo I. Bogotá: Imprenta de Medardo Rivas.

Diario Oficial Extraordinario. (1870). Decreto Orgánico de la Instrucción Pública Primaria. Bogotá: Imprenta de la Nación.

Villamizar, J. (1865). Informe del presidente de Santander a la Asamblea Legislativa de 1865. Socorro: Imprenta de I. Céspedes.

Wilches, S. (1879). Informe del presidente del Estado de Santander a la Asamblea Legislativa en 1879. Socorro: Imprenta del Estado. 


\section{Fuentes secundarias}

Álvarez, A. (1995). ... Y la escuela se hizo necesaria. En busca del sentido actual de la escuela. Santafé de Bogotá: Magisterio-Sociedad Colombiana de Pedagogía.

Báez, M. (2004). Las escuelas normales de varones del siglo xix en Colombia. Historia de la Educación Latinoamericana 6, 179-208. Recuperado de http://redalyc.uaemex.mx/pdf/869/86900611.

Báez, M. (2005). Las escuelas normales colombianas y la formación de maestros en el siglo xix. Eccos-Revista Científica, 2. Recuperado de http:// portal.uninove.br/marketing/cope/pdfs_revistas/eccos/eccos_v7n2/eccosv7n2_2j04.pdf.

De Zubiría, J. (1997). Los modelos pedagógicos. Santafé de Bogotá: Famdi.

Etzioni, A. (1965). Organizaciones modernas. México: Uteha.

González, J. (2005). Legitimidad y cultura. Educación, cultura y política en los Estados Unidos de Colombia, 1863-1886. Bogotá: Universidad Nacional de Colombia.

Herrera, M., y Low, C. (1990). Historia de las escuelas normales en Colombia. Educación y Cultura 20, 41-48.

Jepperson, R. (2001). Instituciones, efectos institucionales e institucionalismo. En W. Powell y P. Dimaggio (comp.). El nuevo institucionalismo en el análisis organizacional (pp. 261-293). México: Colegio Nacional de Ciencias Políticas y Administración Pública-Universidad Autónoma del Estado de México-Fondo de Cultura Económica.

Jepperson, R. y Meyer, J. (2001). El orden público y la construcción de organizaciones formales. En W. Powell y P. Dimaggio (comp.). El nuevo institucionalismo en el análisis organizacional (pp. 261-293). México: Colegio Nacional de Ciencias Políticas y Administración Pública-Universidad Autónoma del Estado de México-Fondo de Cultura Económica.

Loaiza, G. (2007). El maestro de escuela o el ideal liberal de ciudadano en la reforma educativa de 1870. Historia Crítica, 34, 62-91.

Malte, R. (2010). La profesionalización de maestros en la reforma instruccionista de 1870 en el Estado Soberano de Santander. Bucaramanga: Universidad Industrial de Santander.

Powell, W., y Dimaggio P. (2001). El nuevo institucionalismo en el análisis organizacional. México: Colegio Nacional de Ciencias Políticas y Administración Pública-Universidad Autónoma del Estado de México-Fondo de Cultura Económica.

Weber, M. (1974). Economía y sociedad. Esbozo de sociología comprensiva. México: Fondo de Cultura Económica.

Zuluaga, O. (2001). Entre Lancaster y Pestalozzi: los manuales para la formación de maestros en Colombia, 1822-1868. Educación y Pedagogía 29-30, 41-46. 